\title{
La normalización de piedras naturales
}

Fecha de recepción: 31-1-91

RAMON MANIANA VAZQUEZ*

\section{RESUMEN}

El sector de la piedra natural, al igual que cualquier otro sector industrial, requiere el apoyo tecnológico de la normalización para su promoción y desarrollo.

Como consecuencia de la creación de AENOR, se constituyó el CTN 22 "Minería y explosivos", estructurado en siete subcomités. El Subcomité 7 "Piedra natural" está integrado por representantes de los empresarios (Federación de la Piedra), Administración (Dirección General de Minas y de la Construcción, Instituto Tecnológico Geominero de España - ITGE-, Dirección General para la Vivienda y Arquitectura, etc.), Laboratorios (Laboratorio Oficial de Ensayos de Materiales de Construcción - LOEMCO-, Laboratorio Central del MOPU, etc.), Usuarios (Consejo Superior de Colegios de Arquitectos de España, Confederación Nacional de la Construcción).

El Plan de trabajo del SC 7 "Piedra natural" es el siguiente:

- Coordinación de las actividades de los órganos de trabajo del Comité Europeo de Normalización (CEN).

- Organización y coordinación de las actividades del nuevo Comité Técnico de ISO (TC 196 "Piedra natural").

La labor de normalización en el sector de la piedra natural requiere la participación activa en los foros europeos e internacionales de todos los agentes implicados. Para llevar a cabo esta tarea se exige uń intenso esfuerzo de coordinación, tarea asignada a la Secretaría del Comité Técnico de Normalización de Minería y Explosivos desempeñada por AITEMIN.

\author{
$S \cup M M A R Y$
}

The natural stone sector, like any other industrial sector, requires the technological support of standardization for its promotion and development.

As a consequence of the creation of AENOR, CTN 22 "Mining and Explosives" consisting of seven subcommittees, was founded. Subcommittee 7 "Natural Stone" is composed of representatives of the businessmen (Stone Federation), Administration (General Office of Mining and Construction, Tecnnical Geo-Mining Institute of Spain -ITGE-, General Office of Housing and Architecture, etc.), Laboratories (Official Laboratory of Construction Materials Testing - LOEMCO-, Central Laboratory of MOPU (Ministry of Publics Works, etc.), Users (Higher Council of Architectural Colleges of Spain, National Confederation of Construction).

The Work Plan of the SC 7 "Natural Stone" is the following:

- Coordination of the activities of the task groups of the European Committee for Standardization (CEN).

- Organization and coordination of the activities of the new Technical Committee of ISO (TC 196 "Natural Stone").

The work of standardization in the natural stone sector requires the active participation of all the concerned agents in the European and internacional forums. In order to accomplish this task, an intense effort of coordination is required, which is the responsability given to the Secretary of the Technical Committee of Standardization of Mining and Explosives and carried out by AITEMIN.

\section{INTRODUCCIÓN}

El sector de Piedra Natural, al igual que cualquier otro sector industrial, requiere el apoyo tecnológico de la normalización para su promoción y desarrollo.

La existencia de normas en el campo de la piedra natural debe conducir a una serie de beneficios tales como los siguientes:

\section{Extracción}

Racionalización, modernización y tecnificación de las explotaciones mineras de piedra natural, al unificar la maquinaria, técnicas y procedimientos utilizados.

* Director del Departamento de Sistemas Energéticos. E.T.S. de Ingenieros de Minas (U.P.M.)

Vicepresidente de AITEMIN, Presidente del CTN Minería y Explosivos de AENOR.

Presidente del Comité Técnico Internacional ISO/TC 196 "Piedra Natural".

ESPANA. 


\section{Transformación y elaboración}

Mejora de los procesos de tratamiento de piedra natural, a través de un mejor conocimiento de los productos y de sus posibilidades de aplicación, facilitando criterios técnicos y directrices para la transformación y elaboración de los productos, así como para la fabricación de los materiales y maquinaria utilizados en estos procesos.

\section{Comercialización}

Incremento y mejora de la calidad de la oferta nacional de piedras naturales, promoción de los productos mediante "cartas de presentación" a través de catálogos con las especificaciones técnicas de todos los tipos y calidades existentes, mejora de la balanza comercial en este sector evitanto importaciones de calidades que pueden producirse en nuestro país, regulación del mercado facilitando a los fabricantes y usuarios unos criterios técnicos objetivos de valoración de los productos.

\section{ANTECEDENTES EN ESPAÑA}

El Instituto Tecnológico Geominero de España (antes IGME) fue el promotor de un programa de normas de piedra natural. EI ITGE desarrolló esta iniciativa con la colaboración de los productores y transformadores de piedra natural, centros de investigación y laboratorios, representantes de la Administración y la Asociación de Investigación Tecnológica de Equipos Mineros (AITEMIN). Fruto de este programa es la existencia de más de treinta normas UNE, aprobadas por IRANOR, relativas a rocas ornamentales (granitos, mármoles, pizarras, etc.). Esta circunstancia sitúa a España a un nivel de normalización similar e incluso superior al de otros países industrializados.

Las normas UNE 22.170, UNE 22.180 y UNE 22.190 , relativas a características generales de los granitos, mármoles, calizas y pizarras ornamentales, establecen la definición de cada uno de estos tipos de piedra natural, así como la denominación que se les debe aplicar junto a las características que deben reunir.

Los procedimientos de ensayo para evaluar dichas características se desarrollan en un conjunto de normas individuales y específicas para cada grupo de rocas (granitos ornamentales: UNE 22.17122.179, mármoles y calizas ornamentales: UNE 22.181-22.189, pizarras ornamentales: UNE 22.19122.199).

El conjunto de los ensayos establecidos en las normas mencionadas determina un cuadro bastante completo de las características técnicas de cada piedra natural, características que el usuario debe tener en cuenta en el momento de seleccionar un material. Para llegar al establecimiento de los procedimientos de ensayo se tomó como referencia la normativa internacional existente (ASTM, DIN, AFNOR, UNI, BSI, etc.).

\section{SITUACIÓN ACTUAL}

a) En España:

Como consecuencia de la creación de AENOR, se constituyó el CTN 22 "Minería y Explosivos". Dentro de éste está integrado el SC7 "Piedra Natural" cuya composición es la siguiente:

- Federación de Asociaciones para el Desarrollo de la Piedra Natural y Afines (F.D.P.).

- Confederación de Empresarios de Minería y Metalurgia (CONFEDEM).

- Consejo Superior de Colegios de Arquitectos de España.

- Confederación Nacional de la Construcción (CNC).

- Dirección General de Minas y de la Construcción del Ministerio de Industria y Energía. Subdirección General de Industrias de la Construcción.

- Instituto Tecnológico Geominero de España (ITGE).

- Dirección General para la Vivienda y Arquitectura del Ministerio de Obras Públicas y Urbanismo. Subdirección General de Normativa Básica y Tecnología de la Edificación.

- Subdirección General de Minas y Medio Ambiente de la Xunta de Galicia.

- Laboratorio Oficial de Ensayos de Materiales de Construcción (LOEMCO).

- Laboratorio Central (MOPU).

La Presidencia del SC7 la ostenta la FDP y la Secretaría es ejercida por AITEMIN.

El Plan de Trabajo del SC7 consiste en:

- Coordinación de las actividades de los órganos de trabajo del Comité Europeo de Normalización (CEN).

- Organización y coordinación de las actividades del nuevo Comité Técnico de ISO (TC 196 "Piedra Natural").

Para abordar este Plan el SC7 se ha estructurado en 4 Grupos de Trabajo.

En el esquema adjunto se puede ver la estructura y relaciones del SC7 "Piedra Natural" del AEN/CTN 22. 
ESQUEMA DE TRABAJO Y RELACIONES INTERNACIONALES DEL AEN/CTN 22/SC7 "PIEDRA NATURAL"

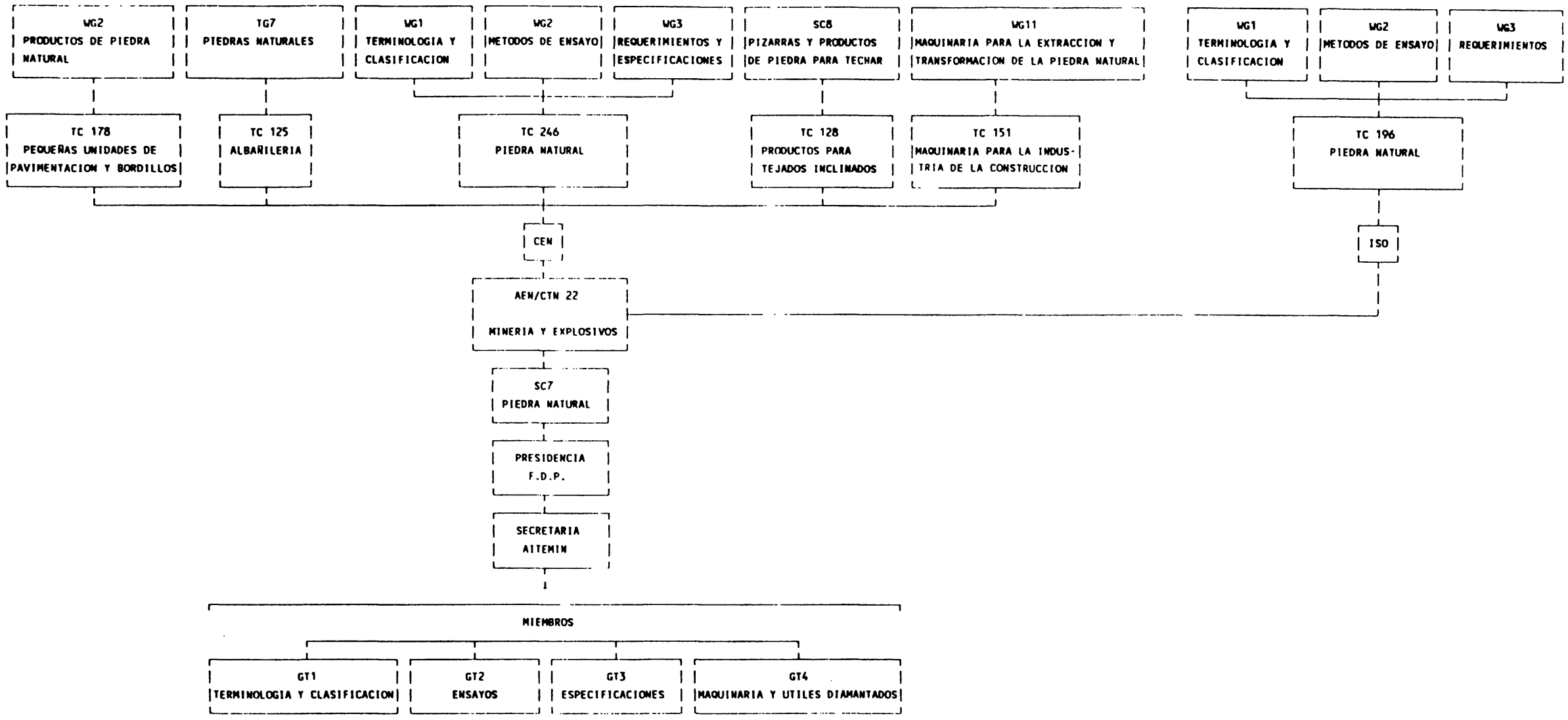




\section{b) En Europa:}

La Directiva comunitaria de 21 de diciembre, relativa a la aproximación de las disposiciones legales, reglamentarias y administrativas de los Estados miembros sobre los productos de construcción (publicada en el Diario Oficial de las Comunidades Europeas de 11 de febrero de 1989) exige que los Estados miembros adopten las disposiciones legales para su aplicación antes del 27 de junio de 1991.

El Artículo 2 de la misma establece que los Estados miembros adoptarán las medidas necesarias para garantizar que los productos de construcción puedan ser comercializados únicamente si son idóneos para el uso a que están destinados, es decir, si cumplen los requisitos básicos relativos a la seguridad, la salud y a otros aspectos de interés colectivo ("requisitos esenciales").

Los requisitos esenciales aplicables a las obras y que pueden influir en las características específicas de un producto son los relativos a los siguientes aspectos:

- Resistencia mecánica (estabilidad).

- Seguridad en caso de incendio.

- Higiene, salud y medioambiente.

— Seguridad de utilización.

— Durabilidad.

- Protección contra el ruido.

- Ahorro de energía.

La Comisión de las Comunidades Europeas ha encargado al Comité Europeo de Normalización (CEN) la elaboración de un programa de normas europeas en el campo de los productos de construcción.

Por lo que respecta a la piedra natural, el CEN ya está desarrollando las actividades de normalización, a través de los siguientes Comités Técnicos, cuyo campo de aplicación afecta a la misma:

- CEN/TC 125 "Albañilería. Productos de construcción".

— CEN/TC 128 "Productos de cubierta para tejados inclinados".

- CEN/TC 151 "Maquinaria para la industria de la construcción".

- CEN/TC 178 "Pequeñas unidades de pavimentación y bordillos".

— CEN/TC 246 "Piedra natural".
El campo de aplicación del CEN/TC 125 es la normalización de los elementos de construcción (en tierra cocida, silicatos de calcio, hormigón, áridos de escoria, etc., hormigones tratados en autoclave) piedras naturales, piedras artificiales, bloques de yeso, morteros y productos artificiales. El órgano de trabajo que está desarrollando las normas específicas de piedras naturales es el WG1/Task Group 7. El documento que están elaborando es el siguiente:

- Proyecto de Norma sobre las piedras masivas y utilizadas en revestimientos delgados verticales.

El campo de aplicación del CEN/TC 128/SC 8 es la normalización en el dominio de las exigencias específicas y métodos de ensayo para pizarras y productos de piedra para techar, designación, terminología, control de las características de los productos. Los documentos que se están elaborando son los siguientes:

- Borrador de Norma Europea: Pizarras naturales y productos de piedra para tejados inclinados. Parte 1. Especificación de producto.

- Borrador de Norma Europea: Pizarras naturales y productos de piedra para tejados inclinados. Parte 2. Métodos de ensayo.

El campo de aplicación del CEN/TC 151 es la normalización en el ámbito de la seguridad de las máquinas y equipos usados en la construcción, siendo el WG11 el encargado de elaborar las normas relativas a las máquinas para la piedra natural.

Por lo que respecta al CEN/TC 178, se definió el campo de aplicación como sigue:

Normalización de las especificaciones de comportamiento y de sus métodos de ensayo asociados para los pequeños elementos rígidos, de revestimiento de suelo, bordillos de acera y los elementos complementarios fabricados en tierra cocida, hormigón o piedra natural para las zonas peatonales, carreteras y otras zonas revestidas, incluyendo las exigencias de puesta en obra. Este comité se ha estructurado en tres grupos de trabajo, siendo el encargado de realizar las normas relativas a la piedra natural el WG2 que se ha dividido en dos "task groups", el TG1 que normaliza los productos de granito y materiales similares y el TG2 que normaliza los productos que no sean de granito.

En cuanto al CEN/TC 246, constituido en septiembre de 1990, su campo de aplicación comprende la definición de los requerimientos, especificaciones y métodos de ensayo para las piedras naturales en forma de bloques en bruto, planchas, productos semiacabados y terminados destinados a ser utilizados en la construcción y para monumentos, con la excepción de aquellos productos que sean tratados por otros comités técnicos. Se han estable- 
cido tres grupos de trabajo cuyos títulos y programas de trabajo se citan a continuación:

WG1. Terminología y clasificación.

- Clasificación petrográfica.

- Definición de nombres comerciales.

- Determinación de las características.

- Terminología para las unidades de piedra para construción y monumentos.

\section{Secretaría: AENOR (España)}

WG2. Métodos de ensayo.

Métodos generales de ensayo relacionados con los materiales y métodos especiales de ensayos relacionados con la aplicación.

\section{Secretaría: AFNOR (Francia)}

WG3. Especificaciones de productos.

Especificación de productos (bloques en bruto, planchas, productos semiacabados y terminados) relacionada con las diferentes aplicaciones.

\section{Secretaría: DIN (Alemania)}

c) Internacional:

En lo que se refiere a normativa internacional, el sector español de la piedra natural (representado por la F.D.P.), a través de AITEMIN y con el apoyo financiero del ITGE, propuso a AENOR la creación de un Comité Técnico Internacional (ISO) en el campo de la piedra natural.

Los días 7 y 8 de noviembre de 1990 tuvo lugar en Madrid la reunión constituyente del ISO/TC 196 "Piedra Natural", siendo elegido como presidente
D. Ramón Mañana Vázquez, y habiéndose aprobado, entre otras, las siguientes resoluciones:

- Campo de aplicación. Normalización en el dominio de las piedras naturales incluyendo la terminología, la clasificación, las exigencias de bloques, planchas, productos semielaborados y terminados, el muestreo, los métodos de ensayo y las exigencias de montaje y puesta en obra.

- Estructura. Se han creado tres grupos de trabajo:

* WG1 Terminología y clasificación.

Secretaría: UNI (Italia).

* WG2 Métodos de ensayo.

Secretaría: AFNOR (Francia).

*WG3 Requerimientos.

Secretaría: AENOR (España).

\section{CONCLUSIÓN}

Tal como se ha puesto de manifiesto, la labor de normalización en el sector de la Piedra natural requiere la participación activa de todos los agentes implicados mediante la defensa de las posturas españolas en los foros europeos e internacionales.

Para llevar a cabo esta labor, se exige un intenso esfuerzo de coordinación. La Secretaría del Comité ISO/TC 196, así como el SC7 "Piedra natural" del CTN Minería y Explosivos de AENOR, y la coordinación con el Comité CEN/TC 246 se conducen por la Secretaría del CTNME, radicada en AITEMIN, C/ Alenza, 1 - 28003 MADRID, Teléfonos: 91/442495566, Fax: 91/4417856.

Sirva esta Nota de presentación y de invitación a todos cuantos se sientan interesados en estas actividades para tomar contacto con esa Secretaría. 